\title{
A new STING-associated monogenic autoinflammatory disease
}

Studies involving a series of children with a distinct vascular and pulmonary syndrome has led to the discovery of an autoinflammatory disease caused by mutations in the gene encoding stimulator of interferon genes protein (STING), a major regulator of interferon signalling. The syndrome, now referred to as STING-associated vasculopathy with onset in infancy (SAVI), is characterized by overproduction of interferon.

The six patients in the study, which was published in New England Journal of Medicine, presented in early infancy with systemic inflammation, cutaneous vasculopathy and pulmonary inflammation. "In children who present with a severe inflammatory disease early in life, the disease is often caused by a monogenic defect with a high penetrance," explains author Raphaela Goldbach-Mansky. "We tested this hypothesis by performing whole-exome sequencing in samples from one patient and her parents, using the latter to filter out genetic variants in the family and to identify a de novo mutation in the patient."
Analysis of the variants revealed such a mutation in TMEM173, which encodes STING, an endoplasmic reticulum transmembrane protein that functions as a homodimer. Evidence of a dysregulated interferon signature in the patient's peripheral blood strongly suggested to the investigators that the mutation might be disease-causing.

Targeted sequencing led to the discovery of de novo mutations in the same gene in a further five unrelated children who had a similar clinical phenotype. Overall, three different mutations were discovered, all located in a small region of exon 5 of TMEM173.

Compared with healthy individuals and patients with another interferonmediated autoinflammatory disease (CANDLE syndrome), patients with SAVI had increased expression of interferonregulated genes in peripheral blood, including the gene encoding IFN- $\beta$ (IFNB1). In these patients, expression of interferon-response genes could not be further upregulated upon stimulation with the STING ligand cyclic GMP-AMP synthase (cGAMP), indicating that the
STING-IFN- $\beta$ pathway is constitutively activated.

Together, the findings suggested the de novo mutations in TMEM173 lead to a gainof-function in STING. "Our data are the first to link gain-of-function de novo mutations in that gene to human disease," reports Goldbach-Mansky.

Treatment options for SAVI are lacking, but the authors demonstrated in lymphocytes from patients that blocking interferon signalling with inhibitors of Janus kinase, such as tofacitinib, ruxolitinib and baricitinib, could have some benefit. "Directly blocking the STING-interferon pathway may emerge as an effective strategy not only in treating SAVI but also other interferon-mediated diseases," suggests Goldbach-Mansky.

\section{Sarah Onuora}

Original article Liu, Y. et al. Activated STING in a vascular and pulmonary syndrome. N. Engl. J. Med. doi:10.1056/ NEJMoa1312625 